\title{
Perfil clínico-epidemiológico dos pacientes internados no pronto-socorro de um hospital universitário
}

\author{
Suzinara Beatriz Soares Lima*, Tânia Solange Bosi de Souza \\ Magnago ${ }^{* \star}$, Ana Cecília Schardong ${ }^{* \star *}$, Roger Rodrigues Peres ${ }^{* \star *}$, \\ Marinez Diniz da Silva Ceron ${ }^{\star \star \star \star *}$, Andrea Prochnow**k*k, Camila \\ de Brum Scalcon ${ }^{\star * \star * * *,}$, Tainara Genro Vieira ${ }^{\star \star * * * * * *}$
}

Resumo: 0 estudo objetivou identificar o perfil clínico-epidemiológico dos pacientes internados no pronto-socorro do Hospital Universitário de Santa Maria, entre agosto de 2008 e julho de 2010. Estudo transversal, descritivo e documental com abordagem quantitativa. Foram avaliados 3.955 atendimentos. Desses, 93,4\% dos pacientes permaneceram internados no pronto-socorro, com uma média de 4,95 dias $( \pm 5,98)$. Predominou o sexo masculino $(59 \%)$, com idade superior a 65 anos $(34,4 \%)$ e procedentes do município de Santa Maria $(61,6 \%)$. A cirurgia geral foi a especialidade médica que obteve maior número de internações. $O$ turno da noite e a quinta feira tiveram maior demanda de internações. Com estes resultados espera-se contribuir na elaboração de soluções de problemas organizacionais, gerenciais, redimensionamento de recursos humanos e previsão de materiais no PS, assim como em estratégias de organização em saúde numa perspectiva ampliada, direcionando olhares e atenções, tão necessárias, a saúde pública.

Descritores: Enfermagem; Serviço hospitalar de emergência; Perfil de saúde.

\section{Clinical and epidemiological profile of patients admitted in the emergency room of a university hospital}

\begin{abstract}
The study aimed to identify the clinical and epidemiological profile of patients admitted in the emergency room of University Hospital of Santa Maria, between August 2008 and July 2010. Crosssectional, descriptive and documental study with a quantitative approach. We evaluated 3955 consultations. Of these, $93,4 \%$ of patients remained admitted in the emergency room, wth na average of 4,95 days $( \pm 5,98)$. Male sex predominant $(59 \%)$, with more than 65 years of age $(34,4 \%)$ and from the city of Santa Maria $(61,6 \%)$. General surgery was the medical specialty that had a bigger number of hospital admissions. The night shift and Thursdays had bigger demands for admissions. With this results, it's expected to contribute on creating solutions to organizational, management, human resources allocation issues and foreknowledge of materials in the ER, as well as strategies on health organizations in an amplified perspective, directing necessary looks and attentions to public health.
\end{abstract}

Descriptors: Nursing; Emergency Service; Hospital; Health profile.

*Pós Doutora pela Universidade Federal de Santa Catarina (UFSC), Florianópolis, SC, Brasil

**Doutora em Enfermagem pela Universidade Federal do Rio de Janeiro (UFRJ), Rio de Janeiro, RJ, Brasil

${ }^{* * *}$ Graduada em Enfermagem pela Universidade Federal de Santa Maria (UFSM), Santa Maria, RS, Brasil

****Mestrando em Enfermagem na Universidade Federal de Santa Maria (UFSM), Santa Maria, RS, Brasil

${ }^{* * * * *}$ Mestrando em Enfermagem na Universidade Federal de Santa Maria (UFSM), Santa Maria, RS, Brasil

****** Mestrando em Enfermagem na Universidade Federal de Santa Maria (UFSM), Santa Maria, RS, Brasil

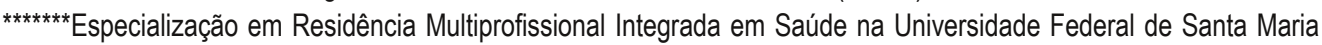

(UFSM), Santa Maria, RS, Brasil

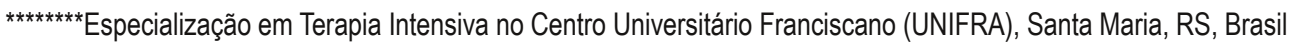




\section{Introdução}

A tecnologia e o progresso científico têm contribuído para o desenvolvimento da área da saúde. No Brasil percebe-se um aumento significativo na quantidade de serviços oferecidos aos pacientes dos sistemas de saúde. Nesses, incluem-se os serviços de pronto-socorro (PS) que enfrentam cotidianamente 0 aumento da demanda por atendimentos. ${ }^{1}$ No entanto, comumente se encontram sem apoio para elucidação diagnóstica e sem articulação com a rede assistencial. ${ }^{2}$

Com relação aos fatores que extrapolam as competências dos pronto-socorros e refletem diretamente na dinâmica de trabalho dos mesmos, pode-se citar 0 baixo investimento em estratégias de promoção da saúde, proteção contra riscos e agentes agressores conhecidos; a ineficaz prevenção de agravos e recuperação e reabilitação da saúde das pessoas. ${ }^{2}$

Nesse sentido, o enfermeiro pode contribuir para a melhoria dessa situação buscando novos instrumentos para organização do trabalho, de modo a promover uma melhora na assistência de enfermagem, além de favorecer a utilização adequada de recursos humanos e materiais para atender as necessidades apresentadas pelos usuários. ${ }^{1}$ Dessa maneira, justifica-se esta pesquisa pela necessidade de conhecer as características clínicas e epidemiológicas das internações realizadas no pronto-socorro, a fim de proporcionar maior preparo e capacitação para a equipe de saúde, em especial a de enfermagem, que atua na unidade em questão. Além disso, acredita-se que tal conhecimento propiciará aos gestores de saúde ferramentas para implementar/implantar ações efetivas que visem a minimização da superlotação das unidades de urgência e emergência.

Esta investigação teve como objetivo identificar o perfil clínico-epidemiológico dos pacientes adultos internados no Pronto-Socorro (PS) do Hospital Universitário de Santa Maria/Rio Grande do Sul (HUSM/RS) no período de agosto de 2008 a julho de 2010.

\section{Metodologia}

Trata-se de uma pesquisa transversal descritiva, retrospectiva com levantamento de dados secundários e abordagem quantitativa. Utilizaram-se os registros eletrônicos dos atendimentos no Pronto-Socorro Adulto (setor de pacientes adultos) do HUSM, no período de agosto de 2008 a julho de 2010.

O local do estudo é classificado como um hospital de grande porte, destinado a prestar assistência nas diversas especialidades médicas, sendo utilizado como campo de ensino, pesquisa e extensão. Esse compreende como área de abrangência a região da quarta coordenadoria regional de saúde do Rio Grande do Sul. O pronto-socorro é composto por três setores de atendimento: "pronto-socorro adulto", "pronto-socorro pediátrico" e "pronto-socorro psiquiátrico". Cada um deles conta com uma estrutura física e com equipes de trabalho específicas. Para este estudo, foram incluídos todos os registros de pacientes do "prontosocorro adulto", que compreende 22 leitos, e excluídos os dos demais setores (pediátrico e

Saúde (Santa Maria), v.39, n.1, p. 77-86, 2013. Perfil clinicoepidemiológico dos pacientes internados no pronto socorro de uma hospital universitário psiquiátrico).

A coleta foi realizada por quatro auxiliares de pesquisa previamente capacitados. Utilizou-se um instrumento desenvolvido com base nos dados presentes nos registros eletrônicos do setor de estatística da instituição pesquisada. 0 instrumento compreende os 
seguintes itens: número de registro do paciente, idade, sexo, procedência, data e hora do atendimento, diagnóstico médico, destino do paciente (alta/óbito/transferência do PS) e data de saída do PS. A partir desses dados foram verificados o dia da semana e o turno do atendimento e calculado o tempo de permanência dos pacientes no pronto-socorro. 0 período de coleta estendeu-se de novembro de 2010 a junho de 2011, pois foi necessário completar os dados faltantes nos registros eletrônicos, acessando os prontuários dos pacientes.

Para a inserção dos dados foi utilizado o programa Excel ${ }^{\circledR}$. Após a verificação de erros e inconsistências, a análise dos dados foi realizada utilizando-se o programa SPSS ${ }^{\circledR} 18.0$ for windows. Utilizou-se a estatística descritiva, com as quais foram construídas médias, medianas, desvio padrão, frequências absolutas e percentagens. Realizaram-se análises bivariadas entre as variáveis estudadas, estabelecendo-se uma significância estatística se $p \leq 0,05$. Os resultados foram apresentados em forma de gráficos e tabelas.

O projeto foi aprovado pelo Comitê de Ética em Pesquisa da Instituição, protocolo n²3081.015279/2010-59, CAAE n. 0264.0.243.000-10 em 08/11/2010.

\section{Resultados e discussão}

Foram coletados dados referentes a 3955 atendimentos realizados no PS adulto do hospital pesquisado, durante o período de 08 de agosto de 2008 a 08 de julho de 2010 . A Tabela 1 apresenta o perfil dos pacientes atendidos.

Tabela 1 - Distribuição dos atendimentos no Pronto-Socorro adulto do Hospital Universitário de Santa Maria, no período de 2008 a 2010. Santa Maria, RS, 2011. (N=3955)

\begin{tabular}{ccc}
\hline Variáveis & $\mathrm{N}$ & $\%$ \\
\hline Sexo (N=3954) & & \\
Masculino & 2332 & 59 \\
Feminino & 1622 & 41 \\
Faixa etária (N=3950) & & \\
12 a 45 anos & 1325 & 33,5 \\
46 a 64 anos & 1266 & 32,1 \\
65 a 107 anos & 1359 & 34,4 \\
Procedência (N=3951) & & \\
Santa Maria & 2434 & 61,6 \\
Outros municípios & 1517 & 38,4 \\
\hline
\end{tabular}

Na Tabela 1, observa-se que há predominância de atendimento a pacientes do sexo masculino (59\%), na faixa etária de 65 a 107 anos (34,4\%) e procedentes do município de Santa Maria $(61,6 \%)$. Na literatura, os estudos revelam maior percentual de atendimentos a Rev. Saúde (Santa Maria), Santa Maria, v.39, n.1, p. 77-86, Jan./Jul.2013. pessoas do sexo feminino. ${ }^{3,4,5}$ 
A predominância do público masculino pode ser refletida a partir das questões culturais e de gênero, pois há vários séculos sustentou-se uma cultura patriarcal em que o homem cultiva a ideia de rejeição da possibilidade de adoecer se expondo mais aos riscos de adoecimento. ${ }^{6}$ Existe a necessidade do homem se desprender das amarras culturais onde acredita ser viril, invulnerável e forte, facilitando assim a adoção de práticas de autocuidado. Pois, caso contrário, procurar o serviço de saúde, numa perspectiva preventiva, poderá associá-lo à fraqueza, medo e insegurança o que, poderia aproximá-lo das representações do universo feminino e implicaria possivelmente em desconfianças acerca dessa masculinidade socialmente instituída. ${ }^{7}$

Destaca-se ainda como provável causa desse número aumentado de atendimentos ao sexo masculino, a maior vulnerabilidade dos homens à violência, na condição de autor ou vítima, gerando traumas e lesões graves devido às agressões. Também, consomem mais bebida alcoólica e tabaco que as mulheres, o que demonstra a maior propensão masculina a doenças cardiovasculares e pulmonares. ${ }^{6}$

Com relação à idade dos pacientes, o maior número de atendimentos ocorreu na faixa etária dos 65 aos 107 anos $(34,4 \% ; N=1359)$ e o menor número de atendimentos se concentrou na faixa etária dos 46 aos 64 anos de idade $(32,1 \% ; N=1266)$. Observa-se divergência nos dados quando comparados a outros estudos realizados, onde constataram maior participação nos atendimentos realizados pelos pronto-socorros para a população jovem, distribuída na faixa dos 18 aos 64 anos de idade., ${ }^{3,5}$

No que tange a procedência, $61,6 \%(\mathrm{~N}=2.434)$ dos pacientes que buscam atendimento no PS são procedentes do município de Santa Maria. Os pacientes encaminhados por outros 81 municípios evidenciam a diversidade de municípios que buscaram atendimento, sendo que $38,4 \%$ ( $N=1517)$ foram realizados por municípios não pertencentes à região de abrangência do hospital, o que pode indicá-lo como referência, não apenas pela localização geográfica, mas pela qualidade da assistência e pela garantia de acesso. ${ }^{8}$

Identificar o município de procedência e a unidade de origem dos pacientes atendidos permite a caracterização do fluxo dos mesmos, favorece o diagnóstico de possiveis distorções e permite a avaliação da pertinência dos encaminhamentos para um serviço hospitalar de alta complexidade. ${ }^{1}$

A partir da constatação de que as portas de urgência constituem-se em importante observatório da condição de saúde da população e da atuação do sistema de saúde, entende-se a importância de identificar a origem dos pacientes que chegam ao serviço. Com o conhecimento destes dados é possível programar melhorias e capacitar as equipes nas unidades e municípios de origem a fim de melhorar a resolubilidade dentro do próprio sistema de saúde. ${ }^{1}$ 
Tabela 2 - Distribuição dos atendimentos no Pronto-Socorro adulto do Hospital Universitário de Santa Maria, no período de 2008 a 2010, segundo características do atendimento. Santa Maria, RS, 2011. (N=3955)

\begin{tabular}{|c|c|c|}
\hline Características do atendimento & $\mathrm{N}$ & $\%$ \\
\hline \multicolumn{3}{|l|}{ Tipo de atendimento } \\
\hline Internação no PS & 3707 & 93,8 \\
\hline Observação & 247 & 6,2 \\
\hline \multicolumn{3}{|l|}{ Permanência do PS (N=3953) } \\
\hline Até 3 dias & 2148 & 54,3 \\
\hline 4 a 117 dias & 1805 & 45,7 \\
\hline \multicolumn{3}{|l|}{ Turno de atendimento $(\mathrm{N}=3954)$} \\
\hline Manhã & 646 & 16,3 \\
\hline Tarde & 1336 & 33,8 \\
\hline Noite & 1972 & 49,9 \\
\hline Atendimentos por dia da semana & $\mathrm{N}$ & $\%$ \\
\hline Segunda feira & 582 & 14,7 \\
\hline Terça feira & 596 & 15,1 \\
\hline Quarta feira & 594 & 15,0 \\
\hline Quinta feira & 670 & 16,9 \\
\hline Sexta Feira & 605 & 15,3 \\
\hline Sábado & 453 & 11,5 \\
\hline Domingo & 454 & 11,5 \\
\hline \multicolumn{3}{|l|}{ Destino do paciente } \\
\hline Transferência & 2154 & 54,5 \\
\hline Alta para casa & 1480 & 37,4 \\
\hline Óbito & 294 & 7,4 \\
\hline Alta por evasão & 20 & 0,5 \\
\hline Alta a pedido & 2 & 0,1 \\
\hline
\end{tabular}

Quanto ao tipo dos atendimentos, maior percentual de pacientes permaneceu internado no pronto-socorro $(93,8 \% ; \mathrm{N}=3707)$. É importante ressaltar que os $37,4 \%(\mathrm{~N}=1.480)$ dos usuários estiveram sob cuidados médicos e da equipe de enfermagem durante 24 horas ou mais, sem necessidade de permanecerem internados, ou foram transferidos para outras unidades ou aos municípios de origem (Tabela 2).

Observa-se na Tabela 2 que 54,3\% ( $N=2148)$ dos pacientes estiveram assistidos pela equipe do pronto-socorro por até três dias (média 4,95; \pm 5,98; mediana=3, mínimo=1 e máximo=117 dias). Embora o maior percentual de pacientes estivesse internado na unidade Rev. Saúde (Santa Maria), Santa Maria, v.39, n.1, p. 77-86, Jan./Jul.2013 por no máximo três dias, nota-se que é alto o número de pessoas internadas por dias, semanas ou até meses que esperam por conduta definitiva ou por leito nas unidades de ${ }^{\text {ISSN }}{ }^{2236-5843}$ 
internação, agravando a problemática da superlotação dessa unidade. Isso é reforçado pelas informações do serviço de estatística do HUSM, que afirma que a taxa de ocupação de leitos no PS nos anos de 2008, 2009 e 2010, foi de 142\%, 140\% e 160\%, respectivamente. ${ }^{9}$

Verifica-se que é alta a taxa de permanência no PS/HUSM quando comparada com outros estudos ${ }^{1,5}$, que teve como tempo de permanência 4 a 30 horas no serviço. A literatura revela que "atualmente, no nosso país, os serviços de emergência funcionam acima de sua capacidade máxima, com taxa de ocupação de leitos acima de 100\%". 10:1

Outro fator que contribui para a baixa rotatividade de leitos no PS avaliado neste estudo foi a localização do setor de traumatologia e ortopedia na mesma unidade. Dez leitos eram destinados a esta clínica, sendo que os pacientes submetidos a procedimentos cirúrgicos retornavam ao PS e ali permaneciam internados até a alta hospitalar. Tal situação desconfigura a característica essencial de uma unidade de emergência, que é a de dar 0 primeiro atendimento e destinar o paciente para uma conduta definitiva em outra unidade. Cabe ressaltar que no final de 2010, o setor de internação em traumatologia e ortopedia foi transferido para a unidade de clínica cirúrgica do hospital em estudo.

De acordo com a Tabela 2, nota-se uma predominância de atendimentos no turno da noite $(49,9 \%)$ seguido pela tarde $(33,8 \%)$ e manhã $(16,6 \%)$. É importante destacar que apesarde evidenciado que as internações ocorrem predominantemente no período da noite, muitosdesses pacientes se encontram em observação no PS desde turnos anteriores, ou até mesmodias, aguardando tratamento definitivo para serem liberados ou permanecerem internados.

O turno que concentra maior número de atendimentos é uma particularidade de cada serviço de pronto-socorro. Estudo ${ }^{3}$ realizado no estado de São Paulo evidenciou que o maior volume de atendimentos ocorreu no período da manhã, ao passo que outro ${ }^{1}$ evidenciou 0 período das 12 às 18 horas com mais atendimentos. Esse é um dado importante para a organização da escala diária de trabalho da unidade, devendo-se direcionar maior número de profissionais aos horários nos quais a demanda é mais elevada. ${ }^{1}$

Quanto ao dia da semana, observa-se que a quinta $(16,9 \%)$, a sexta $(15,3 \%)$, a terça $(15,1 \%)$ e a quarta feira $(15,0 \%)$ foram os dias que tiveram maiores percentuais de atendimento. Esse é outro dado divergente obtido pelo estudo, quando comparado aos resultados de outras pesquisas, que mostram a segunda-feira como 0 dia com maior número de atendimentos. ${ }^{1,3,5}$ De acordo com os estudos, esse dia é o mais procurado devido à facilidade de acesso ao sistema, à disponibilidade de médico sem marcação prévia para atendimento imediato e ao acúmulo de "urgências" não resolvidas no fim de semana. ${ }^{5}$

Quando analisado o destino dos pacientes, verifica-se que $54,5 \%$ foram transferidos para outras unidades de internação dentro do hospital em estudo ou para outras instituições hospitalares. As altas totalizaram 37,4\%; óbitos 7,4\%; alta por evasão $0,5 \%$ e alta a pedido foram dois casos no período avaliado $(0,1 \%)$ - Tabela 2. Das 2.154 transferências, 766 $(35,6 \%)$ usuários foram encaminhados ao Bloco Cirúrgico, $569(26,4 \%)$ transferidos para Unidade de Clínica Médica e 315 (14,6\%) para a Unidade de Clínica Cirúrgica. Dos casos de transferência para outras instituições hospitalares, $22(1 \%)$ pacientes foram encaminhados para hospitais específicos em outros municípios, de acordo com seus diagnósticos médicos e

Saúde (Santa Maria), v.39, n.1, p. 77-86, 2013. Cinicoepidemiologico dos pacientes internados 82 ISSN $2236-5834$ a especialidade atendida naquelas instituições. Este resultado é confirmado em outro estudo ${ }^{1}$ que constata que o principal motivo de alta dos pacientes do pronto-socorro foi a transferênciapara uma unidade de internação hospitalar. 
A distribuição das clínicas especializadas para atendimentos encontra-se na figura 1.

Figura 1 - Distribuição dos atendimentos no pronto-socorro adulto do Hospital Universitário de Santa Maria, segundo clínica especializada, no período de 2008/2010. Santa Maria, RS, 2011. ( $N=3955)$

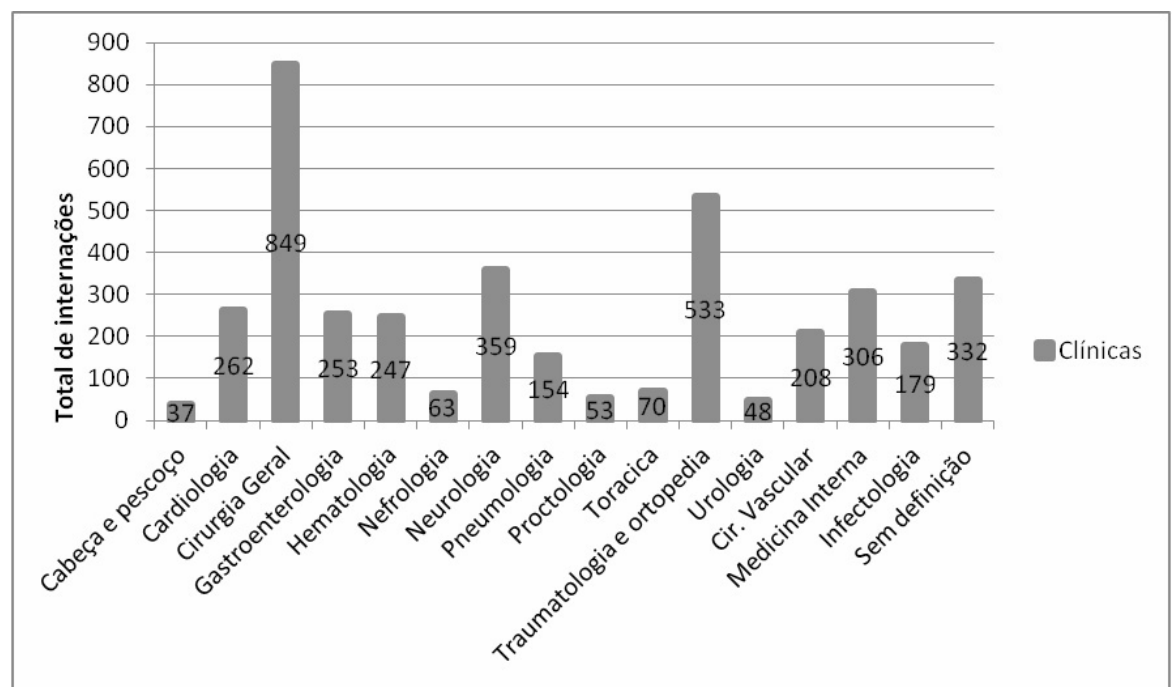

Pela Figura 1, identifica-se 0 alto número de atendimentos pela cirurgia geral $(\mathrm{N}=849)$, seguido pela traumatologia e ortopedia $(\mathrm{N}=533)$, neurologia $(\mathrm{N}=359)$ e de pacientes sem diagnóstico definido que permaneceram sob cuidados da equipe médica e de enfermagem de plantão na unidade ( $\mathrm{N}=332$ ).

A partir da análise da Figura 1, torna-se importante expor os principais diagnósticos encontrados nos atendimentos. Dentre estes, na especialidade da cirurgia geral podem ser citados a litíase biliar, obstrução intestinal, abdome agudo, apendicite aguda e outras doenças do aparelho digestivo, demonstrando o motivo pelo qual a cirurgia geral obteve alto índice de atendimentos no período. Este resultado se encontra compatível com os achados da literatura, que apontam para o fato da apendicite aguda ser um diagnóstico frequente em pacientes que procuram o serviço de emergência, constituindo a causa mais comum de abdome agudo. ${ }^{11}$

Do mesmo modo, as fraturas de membros superiores e inferiores, por quedas, acidentes e agressões merecem destaque. Durante os anos de 2008 a 2010 o serviço de traumatologia do HUSM atendeu no PS, mais de 400 casos de fratura de fêmur devido à queda, principalmente de pessoas idosas. A fratura do fêmur é causa importante de morbidade e mortalidade em idosos, responsabilizando-se por grande parte das cirurgias e ocupação de leitos em enfermarias. ${ }^{12}$ Também, evidenciaram-se neste estudo cerca de 100 casos de fraturas de coluna e outros membros devido a acidentes de trânsito. Nesse caso, 0 alto índice de internação é de pessoas jovens, na maioria das vezes homens na faixa etária de 20 a 39 anos. ${ }^{13}$

Outros diagnósticos relevantes que merecem atenção da equipe médica e de enfermagem são os relacionados a doenças cerebrovasculares. Os acidentes vasculares cerebrais (AVC) obtiveram grande número de internações, assim como os traumas cranioencefálicos. Os quadros neurológicos estão presentes em todas as idades. O AVC destaca-se em número significativo de internações e é uma das causas mais importantes de Rev. Saúde (Santa Maria), Santa Maria, v.39, n.1, p. 77-86, Jan./Jul.2013. mortalidade, hospitalização e incapacidade nas populações desenvolvidas. ${ }^{14}$ 
As doenças cardíacas como o infarto agudo do miocárdio, a insuficiência cardíaca congestiva, as anginas e as arritmias também foram responsáveis por elevado número de atendimentos. Dessa forma, sabe-se que "a hipertensão arterial é a morbidade mais comum na população adulta e frequente nos serviços de emergência, sendo a insuficiência cardíaca a primeira causa cardiovascular de hospitalização no país". 15:1

Podem-se destacar ainda as doenças vasculares (flebites, tromboembolia pulmonar, doença arterial obstrutiva periférica) e as neoplasias, principalmente de pulmão, pâncreas e esôfago. $O$ câncer é um importante problema de saúde pública em países desenvolvidos eem desenvolvimento, onde os tipos de cânceres mais frequentes são os tumores de pulmão, cólon e reto e de estômago. ${ }^{16}$ Esses dados também vão ao encontro dos diagnósticos de neoplasias registrados neste estudo, que evidenciaram o câncer de pulmão como 0 mais expressivo no período analisado.

Destacam-se, ainda, as doenças relacionadas ao vírus da imunodeficiência adquirida (HIV) atendidas pela Infectologia. O HIV atinge milhares de pessoas de diferentes idades e merece destaque nas ações de saúde. Em 2009 foram identificados 3.398 casos de AIDS em jovens de 13 a 24 anos. ${ }^{17}$

Em vias de finalização, destaca-se a partir do estudo realizado e das leituras que deram corpo a discussão, a importância de se conhecer o perfil sociodemográfico e epidemiológico dos usuários da área de abrangência do PS e como esse se articula com as demais instâncias de atenção à saúde, dentro do SUS, no município e seus arredores. ${ }^{1}$

Entende-se que os sistemas regionais devem ter mecanismos efetivos de encaminhamento dos pacientes (referência) aos serviços não disponíveis em seu município/região, como também a garantia de que uma vez atendidas suas necessidades, 0 paciente seja reencaminhado à sua região de origem (contra referência), impedindo, assim, a saturação tão comumente observada nos serviços especializados. ${ }^{2}$

Esta estratégia de organização do sistema de saúde, referência e contra referência, quando bem utilizada pode sanar algumas realidades que são constatadas nos grandes hospitais em que os pacientes se amontoam em macas por falta de leitos hospitalares para sua internação ou ocupam as salas de "emergência" onde permanecem entubados e em ventilação mecânica, por falta de vagas em leitos de terapia intensiva. ${ }^{2}$

Tendo em vista o cenário acima descrito, torna-se imprescindível o requerimento de estratégias governamentais, institucionais e profissionais para adequar o sistema local de saúde na perspectiva das diretrizes da Política Nacional de Atenção às Urgências e do SUS. ${ }^{1}$

Sabe-se que o conhecimento das características da demanda assistida no prontosocorro favorece o trabalho da equipe de saúde. Em unidades complexas, com grande fluxo de atendimentos, transferências, procedimentos e pacientes instáveis é necessário que os profissionais classifiquem as prioridades, cabendo ao enfermeiro, como líder da equipe de enfermagem, organizar e sistematizar a assistência buscando sucesso nas intervenções realizadas.

\section{Conclusão}

Saúde (Santa Maria), v.39, n.1, p. 77-86, 2013. Perfil clínicoepidemiológico dos pacientes internados $84 \quad$ ISSN 2236-5834

O estudo evidenciou que dos 3955 atendimentos registrados no período de 2008 a 2010, $93,8 \%$ ( $N=3707$ ) resultaram em internação hospitalar. Constatou-se que $59 \%$ dos usuários 
atendidos são do sexo masculino com idade superior a 65 anos (34,4\%). Quanto ao tempo de permanência na unidade de emergência, verificou-se que $54,3 \%$ permaneceram por até três dias. $O$ maior percentual dos pacientes foi procedente do município de Santa Maria (61,6\%). A especialidade médica que obteve maior número de internações no período, foi a Cirurgia Geral (849 casos), sendo os diagnósticos de apendicite aguda, fratura do fêmur, neoplasias e doenças cerebrovasculares os mais prevalentes. A quinta feira e o turno da noite foram os que tiveram maior número de atendimentos e internações, respectivamente.

Percebeu-se que os fatores que podem estar relacionados à alta procura por atendimento nos serviços de pronto-socorro podem ser diversos, tendo em vista que estes perpassam por questões como a falta de investimentos nas ações preventivas em saúde, a agilidade do atendimento e o somatório de recursos que os serviços de PS ofertam. Estes transitam em naturezas mais complexas e subjetivas, como por exemplo, questões sociais, epidemiológicas, culturais, geográficas e organizacionais. Com isso a unidade de urgência e emergência frequentemente se desvia do seu objetivo que é 0 de prestar o primeiro atendimento aos casos de urgência/emergência, estabilizar o quadro clínico e transferir a outra unidade para tratamento definitivo.

Entende-se, a partir do estudo, que conhecer a população é fundamental para 0 planejamento, por parte dos administradores federais, estaduais e municipais, e a busca por alternativas que minimizem as dificuldades enfrentadas em serviços de pronto-socorro. A maior rotatividade dos leitos e a existência do serviço de acolhimento e triagem para organizar os atendimentos de acordo com a gravidade, são algumas estratégias para se reduzirem os problemas em unidades de urgência/emergência. Nesse sentido, 0 conhecimento das características da demanda assistida na unidade estudada é imprescindível para que haja sucesso nas intervenções realizadas pela equipe de saúde e, em especial, pela enfermagem, visto que a essa compete a gestão do cuidado em saúde.

Com estes resultados, espera-se ter contribuído para a busca de soluções dos problemas organizacionais e gerenciais, assim como no incentivo a reflexão sobre as estratégias de organização em saúde numa perspectiva ampliada, direcionando olhares e atenções, tão necessárias, a saúde pública.

\section{Agradecimento:}

Agradecemos à Universidade Federal de Santa Maria pelo auxílio Bolsa de Iniciação Científica (FIPE Jr./UFSM/2011).

\section{Referências Bibliográficas}

1. Coelho MF, Chaves LDP, Anselmi ML, Hayashida M, Santos CB. Análise dos aspectos organizacionais de um serviço de urgências clínicas: estudo em um hospital geral do município de Ribeirão Preto, SP, Brasil. Rev Latinoam Enferm. 2010;18(4):[9 telas].

2. Brasil. Ministério da Saúde. Secretaria de Atenção à Saúde. Departamento de Atenção Especializada. Regulação médica das urgências. Editora do Ministério da Saúde: Brasília; 2006.

3. Oliveira GN, Silva MFN, Araujo IEM, Carvalho Filho MA. Perfil da população atendida em uma unidade de emergência referenciada. Rev Latinoam Enferm. 2011;19(3):[09 telas].

Rev. Saúde (Santa Maria), Santa Maria, v.39, n.1, p. 77-86, Jan./Jul.2013. Lima, S.; et al.

4. Silva VPM, Silva AK, Heinisch RH, Heinisch LMM. Caracterização do perfil da demanda da emergência de clínica médica do Hospital Universitário da Universidade Federal de Santa Catarina. ACM Arq Catarin Med. 2007;36(4):18-27. 
5. Jacobs PC, Matos EP. Estudo exploratório dos atendimentos em unidade de emergência em Salvador Bahia. Rev Assoc Med Bras. 2005;51(6):348-53.

6. Brasil. Ministério da Saúde. Secretaria de Atenção à Saúde. Departamento de ações programáticas estratégicas. Política Nacional de Atenção à Saúde do Homem. Brasilia: Editora do Ministério da Saúde; 2008.

7. Gomes R, Nascimento EF, Araújo FC. Por que os homens buscam menos os serviços de saúde do que as mulheres? As explicações de homens com baixa escolaridade e homens com ensino superior. Cad Saúde Pública. 2007;23(3):565-74.

8. Olivati FN, Brandão GAM, Vazquez FL, Paranhos LR, Pereira AC. Perfil da demanda de um pronto-socorro em um município do interior do estado de São Paulo. RFO UPF. 2010;15(3):245-50.

9. Hospital Universitário de Santa Maria (HUSM). Setor de Estatística. Indicadores hospitalares. Santa Maria; 2011.

10. O'Dwyer GO, Oliveira SP, Seta MH. Avaliação dos serviços hospitalares de emergência do programa QualiSUS. Ciênc Saúde Coletiva. 2009;14(5):1881-90.

11. Freire Filho EO, Jesus PEM, D'Ippolito G, Szejnfeld J. Tomografia computadorizada sem contraste intravenoso no abdome agudo: quando e por que usar. Radiol Bras. 2006;39(1):51-62.

12. Cunha U, Veado MAC. Fratura da extremidade proximal do fêmur em idosos: independência funcional e mortalidade em um ano. Rev Bras Ortop. 2006;41(6): 195-9.

13. Confederação Nacional dos Municípios (CNM). Mapeamento das mortes por acidentes de trânsito no Brasil. Brasilia; 2009 [citado em 2011 nov 10]. Disponivel em: http://www.cnm.org.br.

14. Pereira S, Coelho FB, Barros H. Acidente Vascular Cerebral: hospitalização, mortalidade e prognóstico. Acta Med Port. 2004;17:187-92.

15. Lessa I. Epidemiologia da hipertensão arterial sistêmica e da insuficiência cardíaca no Brasil. Rev Bras Hipertens. 2001;8:383-92.

16. Guerra MR, Gallo CVM, Mendonça GAS. Risco de câncer no Brasil: tendências e estudos epidemiológicos mais recentes. Rev Bras Cancerol. 2005;51(3):227-34.

17. Ministério da Saúde (BR). Secretaria de Vigilância em Saúde - Departamento de DST, Aids e Hepatites Virais. Boletim epidemiológico Aids - DST. Brasilia: Editora do Ministério da Saúde; 2010.

\section{Suzinara Beatriz Soares Lima}

Endereço para correspondência - Av. Liberdade, 535. Bairro Passo D'areia, Santa Maria, CEP: 97020490, RS, Brasil.

Email: suzibslima@yahoo.com.br

Currículo Lattes: http://lattes.cnpq.br/6711164523602200

Recebido em 05 de junho de 2013.

Aprovado em 16 de julho de 2013.

Saúde (Santa Maria), v.39, n.1, p. 77-86, 2013. 